\title{
Análisis del ambiente educacional tras la implantación del Plan de Bolonia en la Facultad de Medicina de la Universidad de Zaragoza, España. Comparación con la Facultad de Medicina de la Universidad de Chile
}

\author{
Gabriela Díaz-Véliz, Sergio Mora, Jesús F. Escanero-Marcén
}

Objetivo. Actualmente se acepta que el ambiente educacional influye en forma importante sobre las aptitudes, conocimientos, conducta, logros académicos y grado de satisfacción de los estudiantes. En este trabajo se analizan los cambios en la percepción del ambiente educacional por parte de los estudiantes de la Facultad de Medicina de la Universidad de Zaragoza, como consecuencia de la adaptación de su currículo a las indicaciones del Plan de Bolonia, que comenzó a aplicarse con el primer curso en el año 2009.

Sujetos y métodos. Como instrumento de diagnóstico se aplicó la encuesta de medición de ambiente educacional, conocida como DREEM (Dundee Ready Education Environment Measure), a estudiantes provenientes del primer y tercer curso del año 2008 y a estudiantes del tercer curso del periodo 2011-2012. Como referencia o control, se tomaron las percepciones de los estudiantes de la Facultad de Medicina de la Universidad de Chile durante periodos equivalentes.

Resultados. Con la implantación del Plan de Bolonia, la percepción del ambiente educativo de los estudiantes de la Universidad de Zaragoza ha disminuido significativamente en todos los dominios que forman parte de la encuesta (percepción de la enseñanza, percepción de los docentes, autopercepción académica, percepción del ambiente y autopercepción social).

Conclusión. Los resultados aparentemente negativos de la aplicación del Plan de Bolonia en la Facultad de Medicina de la Universidad de Zaragoza podrían deberse a que el proceso no estaría siendo llevado de la forma mas adecuada por parte de las autoridades y los profesores.

Palabras clave. Ambiente educacional. DREEM. Estudiantes de medicina. Plan de Bolonia. Percepción de estudiantes.

Educational environment analysis after implementation of the Bologna Plan in the Faculty of Medicine of University of Zaragoza, Spain. Comparison with the Faculty of Medicine of the University of Chile

Aim. It is accepted that the educational environment affects aptitudes, knowledge, behaviors, academic achievements and satisfaction degrees among the students. In this study we analyzed the changes of education environment perception declared by the students of the Medical School from the University de Zaragoza, Spain, as a consequence of the curricular changes applied according to the rules established by the Bologna Plan, which begun to be applied in the first degree in 2009.

Subjects and methods. The DREEM questionnaire (Dundee Ready Education Environment Measure) was used to evaluate the educational environment perception among the medical students belonging both to the first and third degree, 2008, and the third degree, 2011-2012. The perception of the medical students of the University of Chile, during similar periods, was taken as reference or control.

Results. After the Bologna Plan application, the education environment perception has significantly decayed among the students of the University of Zaragoza in every of the DREEM questionnaire items (teaching perception, teachers perception, academic self-perception, environment perception and social self-perception).

Conclusions. These apparently negative results of the Bologna Plan in the Zaragoza Medical School could be attributed to an inappropriate conduction by teachers and Faculty members.

Key words. Bologna Plan. DREEM. Educational environment. Medical students. Student perceptions.

Facultad de Medicina; Universidad de Chile; Santiago, Chile (G. DíazVéliz, S. Mora). Facultad de Medicina; Universidad de Zaragoza; Zaragoza, España (J.F. Escanero-Marcén).

Correspondencia:

Profa. Gabriela Díaz Véliz. Programa de Farmacología Molecular y Clínica. Instituto de Ciencias Biomédicas. Facultad de Medicina. Universidad de Chile. P.O. Box 16038. Santiago-9, Chile.

E-mail:

gdiaz@med.uchile.cl

Conflicto de intereses: No declarado.

Competing interests: None declared.

(c) 2013 FEM 


\section{Introducción}

En 1998, la World Federation for Medical Education destacó el medio ambiente educativo como una de las dianas para la evaluación de los programas de educación médica [1]. En la actualidad, está ampliamente aceptado entre los educadores médicos que los efectos del medio ambiente educacional, académico y clínico, son determinantes importantes de las aptitudes, conocimientos, actitudes, progresión y conducta de los estudiantes de medicina [2-4]. El interés por el análisis de las percepciones del medio ambiente educacional (también referido como clima [5]) en las facultades de medicina ha ido en incremento en los últimos años. La calidad del medio ambiente refleja la calidad del currículo [6]. Además, el medio ambiente de aprendizaje es un importante determinante de la conducta [6] y los elementos del medio ambiente educativo se relacionan con los logros académicos y satisfacción en el curso y aspiraciones de los estudiantes [6-10].

La investigación relacionada con el ambiente educacional comenzó en la década de los años treinta y Genn y Harden [5] citan los investigadores más importantes en este dominio hasta los ochenta. Aunque en un principio se estudiaron aspectos relacionados con la 'atmósfera', en las escuelas (primarias y secundarias) y universidades se utilizaron métodos de investigación cualitativa, tales como entrevistas $\mathrm{u}$ observación directa en el interior de las aulas, con el fin de analizar las interacciones entre profesores y alumnos en relación con el entorno físico. Gradualmente, las estrategias de investigación evolucionaron hacia formas de medición cuantitativas con la intención de realizar estudios longitudinales y comparaciones entre instituciones. Hutchins [11] creó uno de los primeros instrumentos específicamente desarrollados para la medición de ambiente educacional en educación médica, el índice de ambiente en escuelas de medicina -Medical School Environment Index (MSEI)-, basándose en el trabajo de Pace y Stern [12], que consistió en un cuestionario de 180 ítems y 18 subescalas o dominios. La Asociación Americana de Escuelas de Medicina (AAMC) utilizó dicha encuesta como parte de un estudio longitudinal realizado en 1956. Dicho estudio permitió distinguir las escuelas/facultades con orientación clínica de las orientadas a la investigación. Además, este instrumento puso de manifiesto algunas de las escuelas de medicina de Estados Unidos que eran percibidas por los alumnos como más agresivas o competitivas [13].
Posteriormente se crearon varias herramientas/ instrumentos de medición del ambiente educacional. Sin embargo, la mayoría quedaron obsoletos tras los profundos cambios conceptuales experimentados en educación médica a partir de la década de los ochenta. Dichos cambios incluyen la promoción del aprendizaje activo y una educación centrada en el estudiante con la introducción del aprendizaje basado en problemas, el aprendizaje durante toda la vida, etc. Estas y otras características son parte del actual Plan de Bolonia [14]. Uno de los instrumentos desarrollados con posterioridad a estos cambios curriculares y que incorpora los conceptos actuales de un currículo innovador es la encuesta de medición de ambiente educacional de Dundee -Dundee Ready Education Environment Measure (DREEM)-, desarrollada por Roff et al en 1997 [15]. Esta encuesta se ha validado en varios países y cuenta con traducciones a diferentes idiomas, incluido el español, que nosotros mismos hemos utilizado en algunas publicaciones [16-18].

El DREEM se ha empleado como medio diagnóstico asociado a otros métodos cualitativos [19] y como metodología única para conocer diferentes aspectos en los centros educativos médicos, los cuales, sin ser exhaustivos, se enumeran a continuación: - Identificar áreas de fortaleza y de debilidad en un medio ambiente educacional normal $[20,21]$.

- Comparar diferentes instituciones educativas médicas $[16,20,22]$.

- Diferenciar las percepciones de estudiantes en diferentes niveles o etapas del curso [20,23,24], entre cursos [25] y género [10,20-22,24-26].

- Detectar zonas de mejora en el currículo [26,27].

- Comparar los alumnos de alto rendimiento con los de bajo rendimiento [10], así como la percepción de alumnos y profesores [28] y de los alumnos en diferentes servicios (diversas especialidades o materias clínicas) [29].

- Realizar estudios multicéntricos comparando el ambiente preclínico en diferentes facultades de medicina [30].

- Medir el medio ambiente educacional existente como línea base para un cambio curricular, con objeto de identificar áreas de prioridad para el cambio y tener un control o referente para la comparación después de realizado el cambio curricular $[24,31]$.

- Comparar el currículo viejo con el nuevo [32,33].

- Investigar/conocer el impacto de un nuevo currículo en el medio ambiente educativo [23,34].

En estos últimos grupos se incluiría el presente trabajo. 
Con el horizonte en el año 2010, todos los centros de las universidades españolas, y entre ellos las facultades de medicina, debían adaptar su currículo a las indicaciones del Plan de Bolonia [14]. En nuestro caso, la Facultad de Medicina de la Universidad de Zaragoza (UZar) comenzó secuencialmente con el primer curso en el año 2009 (curso 2009-2010).

El objetivo de este trabajo consiste en analizar los cambios observados en la percepción del ambiente educacional, por parte de los estudiantes, en los cinco dominios analizados por el DREEM tras la implantación del Plan de Bolonia en los alumnos al final del segundo curso (cuando ya pueden considerarse de tercero), con respecto a la percepción que tenían sus compañeros del plan antiguo. Además se ha tomado otro control o referente: las percepciones de los estudiantes en la Facultad de Medicina de la Universidad de Chile (UCh), con un currículo bastante similar al de la UZar, en el que no ha habido ninguna particular incidencia en los últimos años.

\section{Sujetos y métodos}

\section{Muestra de estudio}

Este trabajo corresponde a un estudio de corte transversal, realizado los años 2008 y 2011. El cuestionario DREEM se aplicó a 676 estudiantes: 344 de la Facultad de Medicina de la UZar y 332 de la Facultad de Medicina de la UCh. La distribución de los estudiantes fue, respectivamente, de 84 y 90 para el primer curso de 2008, 77 y 77 para el tercer curso de 2008, y 171 y 177 para el tercer curso de 20112012. El año 2008 se realizó una medición única en la sala de clases a todos los alumnos presentes de cada curso. El año 2011-2012 se pasó la encuesta un día con actividades obligatorias a fin de obtener un mayor número de respuestas.

En la Facultad de Medicina de la UZar, intencionalmente, se obvió realizar la encuesta a los alumnos del tercer curso, puesto que se trata del curso 'vanguardia', que se enfrenta por primera vez a las innovaciones del cambio curricular y su percepción, públicamente confesada, es altamente negativa. Por ello, y a la luz de los resultados de 2008, donde se estableció una línea base de las percepciones de los alumnos de preclínicas (tres primeros cursos) -véase 'Resultados'-, se pasó el DREEM casi finalizado el segundo curso (muchos alumnos estaban pendientes de una única materia), cuando ellos mismos empiezan a considerarse de tercero.
Ambas facultades seguían currículos tradicionales en el sentido definido por el General Medical Council británico en el año 1993 (currículos centrados en el profesor, basados en disciplinas/materias y en el hospital, con pocas opciones para los estudiantes de elegir diferentes módulos). En el curso 2009-2010, la UZar puso en marcha un nuevo currículo basado en el Plan de Bolonia.

\section{Herramienta utilizada}

El cuestionario DREEM consta de 50 ítems tipo Likert en una escala de 5 puntos ( 0 , muy en desacuerdo; 1 , parcialmente en desacuerdo; 2 , inseguro/dudoso; 3, parcialmente de acuerdo, y 4, totalmente de acuerdo). Debido a que nueve ítems del cuestionario contienen enunciados en negación, se invirtieron sus puntuaciones, de manera que altas puntaciones totales indican una evaluación más positiva en cada aspecto del ambiente educacional.

Los 50 ítems cubren aspectos relevantes para el ambiente educacional y se dividen en cinco dimensiones:

- Dominio 1. Percepción de la enseñanza, con 12 ítems (máx. 48 puntos).

- Dominio 2. Percepción de los docentes, con 11 ítems (máx. 44 puntos).

- Dominio 3. Autopercepción académica, con 8 ítems (máx. 32 puntos).

- Dominio 4. Percepción del ambiente, con 12 ítems (máx. 48 puntos).

- Dominio 5. Autopercepción social, con 7 ítems (máx. 28 puntos).

Al sumar las puntuaciones de los 50 ítems se obtiene un máximo total de 200 puntos. De acuerdo con los resultados obtenidos al sumar las puntuaciones obtenidas por los individuos que respondieron el cuestionario, la puntuación final puede interpretarse de la siguiente manera:

- 0-50 puntos. Ambiente educacional considerado como muy pobre.

- 51-100 puntos. Ambiente educacional con muchos problemas.

- 101-150 puntos. Ambiente educacional con más aspectos positivos que negativos.

- 151-200 puntos. Ambiente educacional excelente.

Para los cinco dominios, según McAleer y Roff [35], las puntuaciones pueden interpretarse del siguiente modo:

- Dominio 1 (percepción de la enseñanza). 0-12, muy pobre; $13-24$, la enseñanza se percibe negativamente; $25-36$, percepción mas bien positiva 
Figura 1. Puntuaciones totales del cuestionario DREEM, expresadas como mediana y rango (mínimo y máximo), según curso y universidad estudiada. UZar: Universidad de Zaragoza; UCh: Universidad de Chile. a $p<$ 0,05 al comparar cursos de una misma universidad.

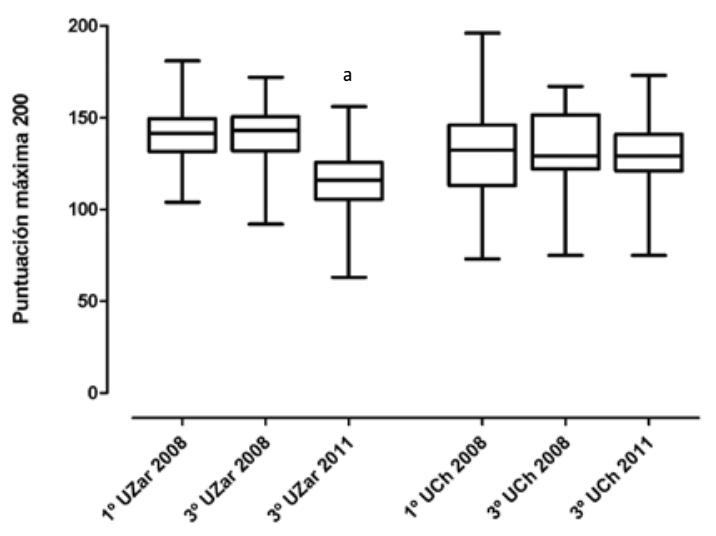

de la enseñanza; 37-48, la enseñanza es muy bien evaluada.

- Dominio 2 (percepción de los docentes). 0-11, abismal (pésimo); 12-22, necesitan entrenamiento educacional; 23-33, encaminados en la dirección correcta; 34-44, docentes modelos.

- Dominio 3 (autopercepción académica). 0-8, sentimiento de fracaso total; 9-16, muchos aspectos negativos; 17-24, sintiéndose más en el lado positivo; 25-32, seguro del futuro académico.

- Dominio 4 (percepción de la atmósfera). 0-11, ambiente pésimo; 12-24, hay muchos aspectos que necesitan cambiar; 25-36, hay una actitud más bien positiva; 37-48, percepción general buena.

- Dominio 5 (autopercepción social). 0-7, miserable; $8-14$, no es un buen lugar; $15-21$, no demasiado mal; 22-28, muy bien socialmente.

El DREEM fue traducido por uno de los autores y posteriormente sometido a un análisis de comprensión por parte del grupo participante, con objeto de utilizar la misma herramienta en los países participantes. Posteriormente se comparó con la versión española del DREEM realizada por Horacio Deza [15] y no se encontraron variaciones que pudieran inducir a interpretaciones diferentes. En aquellos ítems en los que podía utilizarse más de una palabra (p. ej., escuela o facultad), se dio libertad a cada grupo para que emplease ambas o bien la que fuera de uso en su país respectivo, en este caso 'escuela' en Chile y 'facultad' en España. Para otros términos, como 'dictar' o 'explicar', referido a las leccio- nes, también se dio libertad al grupo para utilizar en cada país la más frecuente y la que menos posibilidad de error pudiera suscitar.

\section{Gráficos y tablas}

En España (Zaragoza), los cursos académicos se extienden de septiembre de un año a septiembre del año siguiente. En cambio, en Chile, los cursos académicos coinciden con los años naturales. Para las tablas y gráficos se ha mantenido el año del curso de la UCh; por tanto, éste se refiere al del inicio del curso en la UZar, aunque las pruebas en el caso del año 2008 se realizaron en el primer semestre (antes de navidad). En los alumnos del Plan de Bolonia fue en el segundo semestre, es decir, con el curso de segundo prácticamente concluido, razón por la cual se les ha considerado de tercero.

\section{Tratamiento estadístico}

Para tabular los resultados se calcularon frecuencias, medianas, medias y errores estándares. Para procesar la información los datos se incluyeron en una plantilla de Excel y se analizaron con el programa GraphPad Prism v. 5, utilizando la prueba no paramétrica de Kruskall-Wallis para comparar las puntuaciones de los diferentes cursos de las dos universidades involucradas en este estudio, y la prueba no paramétrica de Dunn para comparaciones múltiples. Se trabajó con un nivel de significación $\alpha=0,05$.

\section{Resultados}

\section{Puntuaciones totales}

Al evaluar las puntuaciones totales en el cuestionario DREEM en las dos universidades estudiadas, sólo se aprecia una disminución significativa en la percepción del ambiente educacional en los estudiantes del curso 2011 de la UZar (Fig. 1).

En la tabla I se observa que en el año 2008 los estudiantes de ambas universidades no mostraron diferencias significativas en las frecuencias relativas a la percepción acerca del ambiente educacional. No obstante, el número de estudiantes que perciben que el ambiente está 'con muchos problemas' en la UCh es mayor que en la UZar, (medias de 9,5\% frente a $0 \%$ y de $9,1 \%$ frente a $1,3 \%$, respectivamente, para los dos cursos estudiados). En las otras tres categorías no hay diferencias significativas.

Por parte de la UZar, los valores han servido para establecer una línea base en los cursos básicos (pre- 
clínicas), dada la estabilidad de los resultados encontrados, y posteriormente contrastarlos en una muestra reducida (21 alumnos) de los alumnos de segundo, donde se repitieron los resultados.

En la misma tabla se observa que, en el año 2011, la categoría 'con muchos problemas' aumentó significativamente en la UZar respecto a lo que se observaba en el año 2008 (21,8\% frente a 1,3\% y 0\%). Además, en la UZar cayó significativamente la apreciación de 'excelente', tanto respecto a las respuestas de los estudiantes de la misma universidad en el año 2008, como en relación a las respuestas de los estudiantes del año 2011 de la UCh. En estos últimos, la apreciación de 'excelente' se mantuvo similar a la manifestada el año 2008.

Puesto que el total se obtuvo sumando las puntuaciones parciales para cada una de las cinco dimensiones, se procede a analizar cada una de ellas con el fin de determinar cuál era más relevante en marcar las diferencias totales observadas entre los cursos de ambas universidades.

\section{Puntuaciones por dominios}

Antes de analizar específicamente las puntuaciones por dominios, hay que hacer notar que los ítems con un promedio inferior a 2,0 se consideran áreas muy problemáticas, mientras que aquellos con un valor superior o igual a 3,5 señalan aspectos realmente positivos, y los ubicados entre 2 y 3 , susceptibles de mejora. De manera general, del total de ítems, el número de los considerados problemáticos y que necesitan intervención fueron, para UZar, 3 , 3 y 14 (primero y tercero con currículo tradicional y tercero con Plan de Bolonia, respectivamente), y para UCh, 4, 4 y 9, respectivamente. En relación a los ítems con 'excelencia' (por encima de 3,5), no existe prácticamente nada a reseñar salvo que en la autopercepción social del estudiante es donde se encuentran en mayor proporción.

\section{Percepción de la enseñanza}

En la figura 2 se evidencia que la percepción de los estudiantes acerca de la enseñanza es muy similar en todos los cursos de ambas universidades, con excepción del curso 2011 de la UZar, en el cual la percepción fue significativamente menor que la de los otros cursos de ambas universidades.

La puntuación de los estudiantes del tercer curso de la UZar (mediana: 21) se encontró en el rango correspondiente a 'la enseñanza es considerada negativamente.' Con el objeto de identificar cuáles eran los aspectos que constituyen debilidades del ambiente educacional y que requieren corregirse, se calculó el
Tabla I. Frecuencia de puntuaciones totales en el cuestionario DREEM.

\begin{tabular}{|c|c|c|c|c|c|c|c|}
\hline \multirow[b]{2}{*}{$\begin{array}{l}\text { Puntuación } \\
\text { total }\end{array}$} & \multirow[b]{2}{*}{$\begin{array}{l}\text { Interpretación del } \\
\text { ambiente educacional }\end{array}$} & \multicolumn{3}{|c|}{ Universidad de Zaragoza } & \multicolumn{3}{|c|}{ Universidad de Chile } \\
\hline & & $\begin{array}{c}1.9 \\
2008\end{array}$ & $\begin{array}{c}3.9 \\
2008\end{array}$ & $\begin{array}{c}3.9 \\
2011\end{array}$ & $\begin{array}{c}1.9 \\
2008\end{array}$ & $\begin{array}{c}3.9 \\
2008\end{array}$ & $\begin{array}{l}3.9 \\
2011\end{array}$ \\
\hline$<50$ & Muy pobre & $0,0 \%$ & $0,0 \%$ & $2,3 \%$ & $0,0 \%$ & $0,0 \%$ & $0,0 \%$ \\
\hline $51-100$ & Con muchos problemas & $0,0 \%$ & $1,3 \%$ & $21,8 \%^{a}$ & $9,5 \%$ & $9,1 \%$ & $13,7 \%$ \\
\hline $101-149$ & Más positivo que negativo & $75,6 \%$ & $74,0 \%$ & $74,7 \%$ & $71,4 \%$ & $63,6 \%$ & $67,1 \%$ \\
\hline $150-200$ & Excelente & $24,4 \%$ & $24,7 \%$ & $1,2 \%$ a & $19,1 \%$ & $27,3 \%$ & $19,2 \%$ \\
\hline
\end{tabular}

${ }^{a} p<0,05$ respecto a los otros cursos de la misma universidad.

promedio de respuestas de cada uno de los ítems de este dominio. Según la percepción de los estudiantes del curso 2011 de la UZar, casi todos los ítems correspondientes a este dominio obtuvieron valores preocupantes (Tabla II). Hay que señalar que los dos últimos ítems son de aquellos que requieren una codificación inversa. Además, se aprecia que en este dominio no se observaron ítems con valores mayores a 3,5 (excelentes) en ninguno de los cursos de este estudio.

\section{Percepción de los docentes}

En este dominio, los estudiantes del curso 2011 de la UZar fueron los que tuvieron significativamente más problemas en esta universidad (Fig. 3), con descensos en las puntuaciones con respecto a los del curso 2008. Sin embargo, todos los cursos de ambas universidades quedaron en el rango 23-33 ('encaminados en la dirección correcta'), incluso el curso 2011 de la UZar, con una mediana de 25.

Casi todos los ítems de este dominio mostraron valores intermedios, aunque dos cursos de la UZar, uno del 2008 y otro del 2011, calificaron con una puntuación inferior a 2,0 los ítems 'los docentes se molestan y alteran en clases' y 'los docentes son autoritarios' (Tabla III). Un ítem muy bien calificado $(3,0$ a 3,3$)$ por todos los estudiantes encuestados fue 'los docentes están bien preparados para sus clases'. Sin embargo, en la UZar se puede observar un par de ítems que el año 2011 bajaron la buena apreciación que habían tenido el año 2008, aunque se mantienen por encima de 2,0 ('los docentes dan ejemplos claros' y 'los docentes conocen la materia que dictan').

\section{Autopercepción académica}

Se aprecia que los estudiantes del curso 2011 de la UZar tuvieron significativamente la peor autoper- 
Tabla II. Dominio 1: percepción del estudiante acerca de la enseñanza.

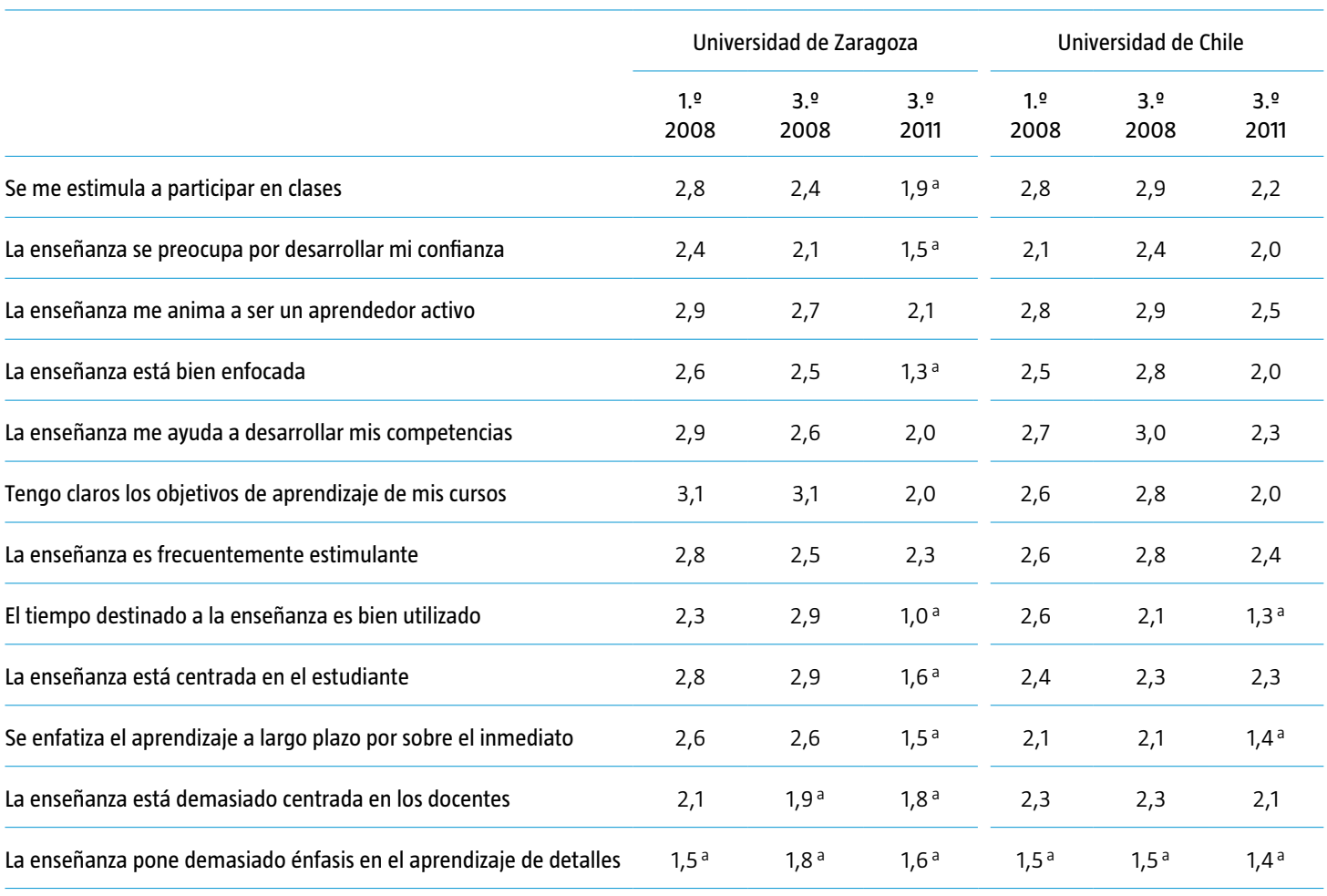

a Ítems preocupantes.

Figura 2. Percepción de la enseñanza, según curso y universidad estudiada, expresada como mediana y rango (mínimo y máximo). UZar: Universidad de Zaragoza; UCh: Universidad de Chile. a $p<0,05$ al comparar cursos de una misma universidad.

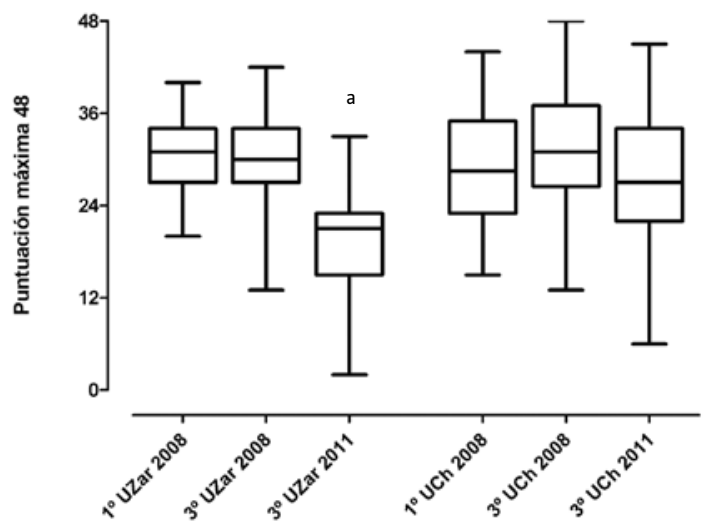

cepción dentro de su universidad (Fig. 4), aunque todos los cursos de ambas universidades quedaron dentro del rango de 17-24 ('sintiéndome más en el lado positivo').

En general, en este dominio no se observaron ítems preocupantes y aparece una calificación superior a 3,0 en el ítem 'tengo la confianza que voy a aprobar este curso' en todos los cursos de ambas universidades (Tabla IV). Sin embargo, llaman poderosamente la atención y no dejan de ser alarmantes los importantes descensos en los siguientes ítems: 'siento que me están preparando bien para mi profesión,' 'lo aprendido el año pasado fue una buena base para el trabajo de este año, 'la escuela/facultad me ayuda a desarrollar mis destrezas para resolver problemas' y 'he aprendido mucho sobre la empatía en mi profesión,' observados en el curso 2011 de la UZar y que no ocurren en el curso 2011 de la UCh. En esta última, un ítem que oscila alrededor de lo preocupante en todos los cursos es 'los métodos de estudio que tenía antes todavía me sirven'.

\section{Percepción del ambiente de aprendizaje}

En la figura 5 se aprecia que los estudiantes del primer curso de la UCh del año 2008 se mostraron significativamente más satisfechos que los estudiantes 
Tabla III. Dominio 2: percepción que tiene el estudiante acerca de los docentes.

\begin{tabular}{|c|c|c|c|c|c|c|}
\hline & \multicolumn{3}{|c|}{ Universidad de Zaragoza } & \multicolumn{3}{|c|}{ Universidad de Chile } \\
\hline & $\begin{array}{c}1.9 \\
2008\end{array}$ & $\begin{array}{c}3 . . \\
2008\end{array}$ & $\begin{array}{c}3.0 \\
2011\end{array}$ & $\begin{array}{c}1.0 \\
2008\end{array}$ & $\begin{array}{c}3 . . \\
2008\end{array}$ & $\begin{array}{c}3.0 \\
2011\end{array}$ \\
\hline Los docentes son buenos dando retroalimentación a los estudiantes & 2,6 & 2,5 & 2,0 & 2,7 & 2,8 & 2,4 \\
\hline Los docentes tienen buenas destrezas comunicacionales con los pacientes & 2,5 & 2,8 & 2,3 & 2,8 & 3,0 & 3,1 \\
\hline Los docentes están bien preparados para sus clases & 3,0 & 3,1 & 3,0 & 3,3 & 3,1 & 3,1 \\
\hline Los docentes dan ejemplos claros & 3,0 & 3,0 & 2,5 & 3,1 & 3,1 & 3,1 \\
\hline Los docentes conocen las materias que dictan & 3,0 & 3,2 & 2,8 & 3,3 & 3,5 & 3,4 \\
\hline En la escuela/facultad, los docentes nos hacen críticas constructivas & 2,6 & 2,6 & 2,2 & 2.6 & 2,9 & 2,7 \\
\hline Los docentes ridiculizan a los estudiantes & 2,8 & 3,1 & 2,9 & 2,6 & 2,7 & 2,9 \\
\hline Los docentes se molestan y alteran en clases & $1,9^{a}$ & 2,4 & $1,9^{\mathrm{a}}$ & 2,2 & 2,8 & 2,9 \\
\hline Los docentes son autoritarios & $1,7^{\mathrm{a}}$ & 2,2 & $1,9^{\mathrm{a}}$ & 2,2 & 2,3 & 2,4 \\
\hline Los docentes tienen paciencia con los pacientes & 2,5 & 2,7 & 2,5 & 2,6 & 2,7 & 2,9 \\
\hline Los estudiantes causamos irritación a los docentes & 2,2 & 2,8 & 2,2 & 2,2 & 2,7 & 2,8 \\
\hline
\end{tabular}

a Ítems preocupantes.

del curso superior, tanto del año 2008 como del 2011, en tanto que en la UZar se observa que la buena percepción del año 2008 cae significativamente el año 2011. En esta universidad, los estudiantes de ambos cursos del año 2008 calificaron este dominio en el rango de 'una percepción general buena', la cual cae a 'una actitud más bien positiva' el año 2011. Sin embargo, esta percepción es similar a la observada en todos los cursos de la UCh.

En la tabla V se aprecia que en cuatro de los ítems coincide la buena apreciación de los estudiantes de todos los cursos de ambas universidades (rango: 2,9$3,5)$, mientras que en el resto de los ítems se observa una disminución de la apreciación en el curso del 2011. Un ítem que muestra resultados preocupantes en algunos cursos de ambas universidades es el que se refiere a 'los horarios de la escuela están bien programados', y otro de urgente análisis, 'en esta escuela/facultad la copia en las pruebas constituye un problema'. En otros ítems, aunque la puntuación no es alarmante, se puede apreciar la preocupación que están manifestando los estudiantes. Se trata de 'el ambiente de la escuela/facultad me motiva a aprender' y 'el ambiente de aprendizaje es agradable'. El ítem 'el ambiente durante las clases teóricas es rela-
Figura 3. Percepción de los docentes, según curso y universidad estudiada, expresada como mediana y rango (mínimo y máximo). UZar: Universidad de Zaragoza; UCh: Universidad de Chile. ${ }^{\text {a }} p<0,05$ al comparar cursos de una misma universidad.

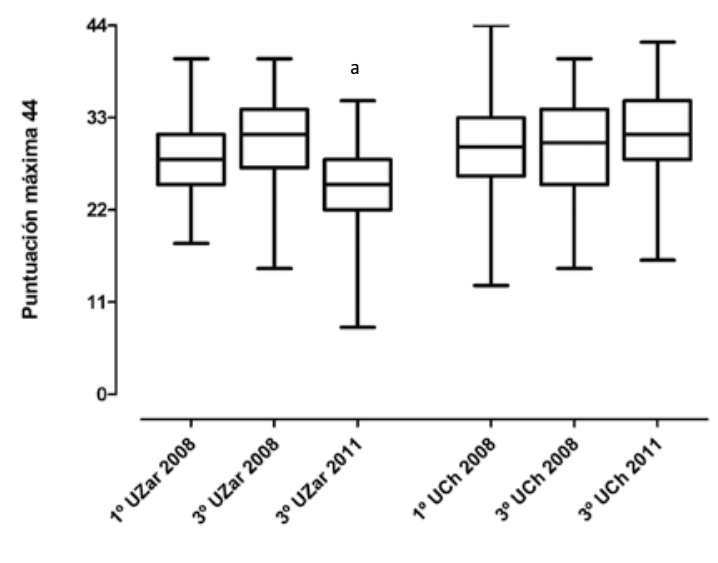

jado' muestra una caída el año 2011 en la UZar; en cambio, en la UCh, muestra un nivel bajo en todos los cursos encuestados. 
Tabla IV. Dominio 3: autopercepción académica del estudiante.

\begin{tabular}{|c|c|c|c|c|c|c|}
\hline & \multicolumn{3}{|c|}{ Universidad de Zaragoza } & \multicolumn{3}{|c|}{ Universidad de Chile } \\
\hline & $\begin{array}{c}1.9 \\
2008\end{array}$ & $\begin{array}{c}3.9 \\
2008\end{array}$ & $\begin{array}{c}3 . . \\
2011\end{array}$ & $\begin{array}{c}1.0 \\
2008\end{array}$ & $\begin{array}{c}3.9 \\
2008\end{array}$ & $\begin{array}{l}3 . . \\
2011\end{array}$ \\
\hline Soy capaz de memorizar todo lo que me es necesario & 3,1 & 3,0 & 2,8 & 2,2 & 2,1 & $1,8^{a}$ \\
\hline $\begin{array}{l}\text { Mucho de lo que tengo que aprender me } \\
\text { parece relevante en mi carrera como médico }\end{array}$ & 2,7 & 2,8 & 2,6 & 2,7 & 2,8 & 2,2 \\
\hline Siento que me están formando bien para mi profesión & 3,0 & 2,9 & 2,2 & 3,3 & 3,1 & 2,7 \\
\hline $\begin{array}{l}\text { Lo aprendido el año pasado fue una } \\
\text { buena base para el trabajo de este año }\end{array}$ & 2,9 & 2,9 & 2,3 & 2,0 & 3,0 & 2,8 \\
\hline $\begin{array}{l}\text { La escuela/facultad me ayuda a desarrollar } \\
\text { mis destrezas para resolver problemas }\end{array}$ & 2,7 & 2,8 & 2,2 & 2,8 & 2,9 & 2,7 \\
\hline Tengo la confianza que voy a aprobar este año & 3,6 & 3,4 & 3,4 & 3,3 & 3,3 & 3,1 \\
\hline He aprendido mucho acerca de empatía en mi profesión & 2,6 & 2,9 & 2,0 & 3,0 & 3,2 & 3,0 \\
\hline Los métodos de estudio que tenía antes todavía me sirven & 2,9 & 3,2 & 2,8 & $1,8^{\text {a }}$ & 2,4 & 2,0 \\
\hline
\end{tabular}

a Ítems preocupantes.

Figura 4. Autopercepción académica, según curso y universidad estudiada, expresada como mediana y rango (mínimo y máximo). UZar: Universidad de Zaragoza; UCh: Universidad de Chile. a $p<0,05$ al comparar cursos de una misma universidad.

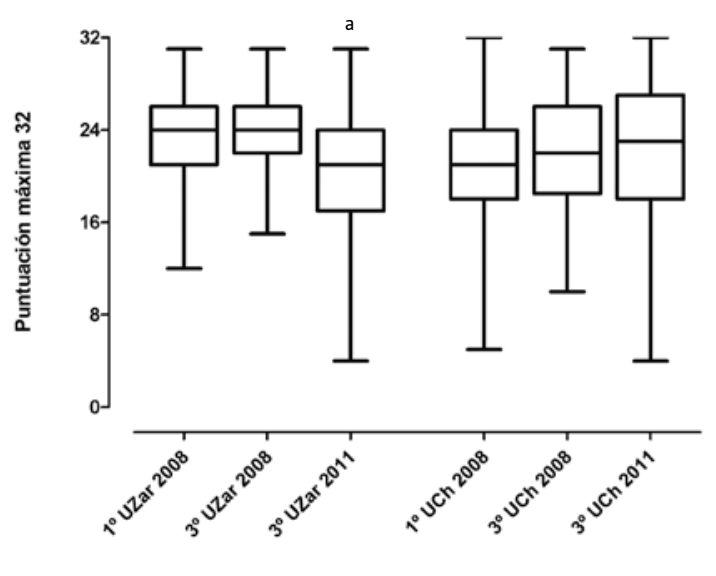

\section{Autopercepción social}

Respecto a la autopercepción social (Fig. 6), no se observan diferencias significativas en las puntuaciones medias de los tres cursos de la UCh, quedando en el rango 'no demasiado mal'. En la UZar no se apreciaron diferencias significativas entre los cursos del 2008, pero esta apreciación cayó significativamente el año 2011.

Los estudiantes de ambas universidades tuvieron una excelente percepción en varios de los ítems de este dominio (Tabla VI), aunque algunos otros mostraron puntuaciones preocupantes, como hay un buen sistema de apoyo para los estudiantes que sufren estrés' y 'estoy demasiado cansado para disfrutar los cursos que estoy tomando'. También destaca la baja puntuación encontrada en el ítem 'rara vez me aburro en las clases' en ambas universidades.

\section{Discusión}

Los resultados globales presentados permiten obtener una importante conclusión: la percepción global del ambiente educativo de los estudiantes españoles ha disminuido significativamente respecto de la que tenían sus compañeros cuando se desarrollaba el anterior currículo. Genn [6] indicó que la calidad del medio ambiente educacional refleja la calidad del currículo y, en consecuencia, los niveles de satisfacción de los estudiantes, de aprendizaje y de éxito o logro. En este sentido, y derivados de los resultados globales, nadie, ni profesorado ni responsables del gobierno de la facultad/universidad, pueden estar satisfechos con los resultados que se presentan en este trabajo. 
Tabla V. Dominio 4: percepción del estudiante acerca del ambiente de aprendizaje.

\begin{tabular}{|c|c|c|c|c|c|c|}
\hline & \multicolumn{3}{|c|}{ Universidad de Zaragoza } & \multicolumn{3}{|c|}{ Universidad de Chile } \\
\hline & $\begin{array}{c}1.0 \\
2008\end{array}$ & $\begin{array}{c}3 .{ }^{\circ} \\
2008\end{array}$ & $\begin{array}{c}3.0 \\
2011\end{array}$ & $\begin{array}{c}1.0 \\
2008\end{array}$ & $\begin{array}{c}3.0 \\
2008\end{array}$ & $\begin{array}{c}3.0 \\
2011\end{array}$ \\
\hline El ambiente durante las clases teóricas es relajado & 3,5 & 3,3 & 2,6 & 2,4 & 2,6 & 2,1 \\
\hline Siento que puedo preguntar todo lo que quiero & 3,1 & 3,3 & 3,0 & 3,1 & 3,0 & 3,1 \\
\hline Me siento socialmente cómodo en clases & 3,4 & 3,4 & 3,3 & 3,2 & 3,1 & 3,3 \\
\hline Tengo oportunidades para desarrollar habilidades interpersonales & 3,3 & 3,3 & 3,0 & 3,0 & 2,9 & 2,9 \\
\hline El ambiente durante los seminarios y trabajos tutoriales es relajado & 3,3 & 3,0 & 2,9 & 2,9 & 3,0 & 2,9 \\
\hline El placer de estudiar medicina es mayor que el estrés que esto me produce & 2,8 & 2,4 & 2,5 & 2,9 & 2,3 & $1,9^{\mathrm{a}}$ \\
\hline El ambiente de la escuela/facultad me motiva a aprender & 3,0 & 2,8 & 2,4 & 2,9 & 2,4 & 2,2 \\
\hline Soy capaz de concentrarme adecuadamente & 3,0 & 3,0 & 2,8 & 2,3 & 2,3 & 2,1 \\
\hline El ambiente de aprendizaje es agradable & 3,4 & 3,2 & 2,7 & 2,9 & 2,7 & 2,3 \\
\hline Los horarios de aprendizaje en la escuela/facultad están bien programados & 2,6 & 2,9 & $1,2^{\mathrm{a}}$ & 2,4 & $1,3^{\text {a }}$ & $1,0^{\mathrm{a}}$ \\
\hline Mi experiencia de aprendizaje en la escuela/facultad ha sido desalentadora & 3,5 & 3,3 & 2,8 & 3,1 & 3,0 & 2,8 \\
\hline En esta escuela/facultad la copia en las pruebas constituye un problema & 2,9 & 3,0 & 2,0 & 2,2 & 2,3 & 2,3 \\
\hline
\end{tabular}

Los resultados para los alumnos de primer y tercer curso que siguen un currículo tradicional en la UZar coinciden sensiblemente. Si además se tiene en cuenta que 21 alumnos de segundo curso que voluntariamente realizaron el DREEM proporcionaron valores medios coincidentes con los resultados comunicados, los valores presentados en este trabajo se han considerado una auténtica línea base para los estudiantes de preclínicas. Con los resultados de la UCh podría ocurrir un hecho similar que resulta contrastado, con pequeños matices, con los resultados obtenidos por los alumnos de tercero en 2011. Es interesante tener en cuenta estas pequeñas matizaciones porque dicho comportamiento va a servir de línea base con objeto de corroborar que el estudio transversal es válido en el sentido de que el paso de unos años (tres en concreto) no representó variación en los valores de la línea base.

Los resultados globales del trabajo, tanto para la Uzar como para la UCh, concuerdan con muchas de las facultades de medicina de nuestro entorno, como ya se ha discutido en publicaciones anteriores [16-18]. Más concretamente, debe indicarse que la mayoría de las publicaciones comunican valores
Figura 5. Percepción del ambiente de aprendizaje, según curso y universidad estudiada, expresada como mediana y rango (mínimo y máximo). UZar: Universidad de Zaragoza; UCh: Universidad de Chile. ${ }^{a} p<0,05$ al comparar cursos de una misma universidad.

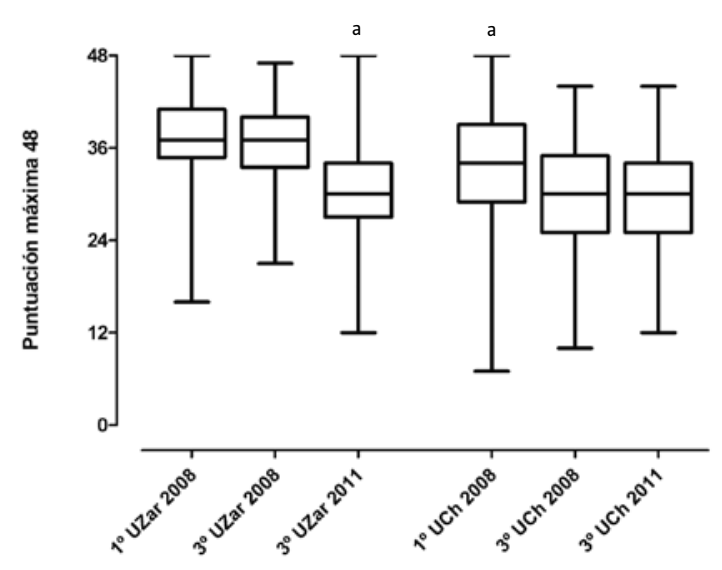

con ambientes educacionales 'con más aspectos positivos que negativos' [35] (100-150 puntos, del 50 
Tabla VI. Dominio 5: autopercepción social del estudiante.

\begin{tabular}{|c|c|c|c|c|c|c|}
\hline & \multicolumn{3}{|c|}{ Universidad de Zaragoza } & \multicolumn{3}{|c|}{ Universidad de Chile } \\
\hline & $\begin{array}{c}1.0 \\
2008\end{array}$ & $\begin{array}{c}\text { 3.o } \\
2008\end{array}$ & $\begin{array}{c}3.0 \\
2011\end{array}$ & $\begin{array}{c}1.0 \\
2008\end{array}$ & $\begin{array}{c}3 . .9 \\
2008\end{array}$ & $\begin{array}{l}3 . \circ \\
2011\end{array}$ \\
\hline Tengo buenos amigos en la escuela/facultad & 3,7 & 3,6 & 3,7 & 3,7 & 3,5 & 3,7 \\
\hline Hay un buen sistema de apoyo para los estudiantes que sufren estrés & 2,1 & $1,7^{\mathrm{a}}$ & $1,4^{\mathrm{a}}$ & $1,8^{a}$ & $1,3^{a}$ & $1,3^{\circ}$ \\
\hline Estoy demasiado cansado para disfrutar los cursos que estoy tomando & 2,7 & 2,6 & $1,8^{\mathrm{a}}$ & $1,8^{a}$ & $1,1^{\mathrm{a}}$ & $1,1^{\mathrm{a}}$ \\
\hline Rara vez me aburro en las clases & 2,3 & 2,2 & $1,8^{\mathrm{a}}$ & 2,1 & 2,2 & $1,4^{2}$ \\
\hline Los ambientes físicos de la escuela/facultad son agradables & 3,7 & 3,8 & 3,7 & 3,3 & 3,4 & 3,4 \\
\hline Mi vida social es buena & 3,7 & 3,5 & 3,4 & 3,2 & 3,0 & 3,0 \\
\hline Rara vez me siento solo & 3,3 & 3,2 & 3,1 & 2,8 & 2,8 & 2,8 \\
\hline
\end{tabular}

Figura 6. Autopercepción social, según curso y universidad estudiada, expresada como mediana y rango (mínimo y máximo). UZar: Universidad de Zaragoza; UCh: Universidad de Chile. a $p<0,05$ al comparar cursos de una misma universidad.

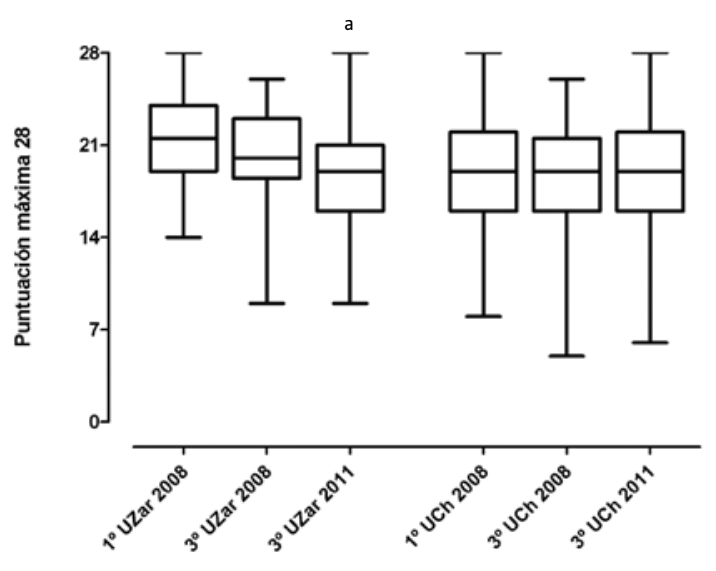

al $75 \%$ de la puntuación), con algunas excepciones: una por encima [29], con valores del 85\% (DREEM modificado), y tres por debajo del $50 \%[23,27,36]$, a las que podrían añadirse otras [31,33] con valores precisos del $50 \%$. Sin embargo, el rango del $50-75 \%$ es muy amplio y existen claras diferencias entre los valores de la bibliografía. Los valores de este trabajo se hallan más en consonancia con los valores ubicados en la mitad superior del rango [19,20,21,25,31,33 -cu- rrículum híbrido con Plan de Bolonia-, 37,38] que en la mitad inferior $[10,20,26,28,31,33,39,40]$.

En el párrafo anterior se han omitido intencionadamente las publicaciones realizadas en Chile sobre el DREEM, con el objetivo de efectuar un comentario aparte. En un estudio multicéntrico [30] para analizar el ambiente preclínico de seis escuelas de medicina (entre las cuales no se encontraba la UCh), realizado el mismo año que los datos de este estudio (2008), los resultados para primer y tercer curso de la UCh $-131,1(65,6 \%)$ y $130,7(65,4 \%)$, respectivamente- podrían considerarse en el nivel de las escuelas señaladas en las primeras posiciones, ya que las otras obtienen puntuaciones considerablemente más bajas. De forma más específica, en otro estudio acerca del ambiente curricular [34] realizado en la Pontificia Universidad Católica de Chile para medir la percepción de los estudiantes del nuevo currículo, el resultado promedio para estudiantes de tercer a quinto año fue de 127,5 (63,8\%), lo que coincide sensiblemente con los aportados en este estudio. Desconocemos que en España se haya publicado algo al respecto, salvo nuestros datos [17]. Sin embargo, los datos comunicados anteriormente no concuerdan con la opinión de Roff [41], quien resumiendo la bibliografía internacional indicó que las escuelas tradicionales generalmente puntúan menos de 120 puntos (60\%).

Antes de proseguir con la discusión de los resultados comunicados en los diferentes dominios se deben advertir, para su justa comprensión, los dos hechos siguientes: uno, el cuestionario utilizado no 
proporciona las razones subyacentes que expliquen las respuestas de los estudiantes, y otro, como indica Till [23], este cuestionario se ha utilizado para un objetivo muy concreto pero no presenta el tratamiento a seguir para modificar en el sentido adecuado las alteraciones encontradas en el ambiente educativo tras la imposición del Plan de Bolonia en la Facultad de Medicina de la UZar. No obstante, tras la constatación de los hechos, no podemos sustraernos a deslizar algunas sugerencias en el sentido de su posible corrección [16,18,33,41].

Con la implantación del Plan de Bolonia, el ambiente educacional en la UZar cayó significativamente, de $140 \%$ a $114 \%$, decreciendo la puntuación en todos los dominios. En el dominio 1, la caída resultó tremendamente llamativa porque pasó de tener uno y dos ítems con puntuación inferior a 2 en primero y tercero del 2008, a ocho ítems en el año 2011. Los cuatro ítems que mantienen una puntuación de dos o ligeramente superior se relacionan con una valoración general de la enseñanza, mientras que el resto valora aspectos más puntuales de ésta. Todos ellos se encuentran en un rango de 1,51,9 , destacando como muy inferiores 'la enseñanza está bien enfocada' $(1,3)$ y 'el tiempo destinado a la enseñanza esta bien utilizado’ $(1,0)$. Estos resultados discrepan notablemente de los comunicados en otros trabajos. En este sentido, Whittle et al [19] señalaron que, en la Universidad de Leeds, en todo el cuestionario obtuvieron tres ítems con puntuaciones inferiores a 2, de los cuales solamente coincidimos en uno y es referente a la autopercepción social: 'hay un buen sistema de apoyo para los estudiantes que sufren estrés'. Su manera de afrontar el problema puede ser un indicador de un correcto tratamiento. Tras el análisis del mismo se procedió a la instalación, en todos los centros de enseñanza, de carteles que indicaban el camino a seguir en caso de requerir apoyo y el personal encargado de brindarlo. El problema ocurría por una falta de conocimiento por parte de los estudiantes de los servicios disponibles en la universidad. Algo similar sucede en la UZar. En cambio, en la UCh existe un programa de tutorías para brindar apoyo y orientación a los estudiantes que lo requieren.

La mala utilización del tiempo destinado a la enseñanza no es un problema reciente y constituye un indicativo claro de que no están optimizadas las estrategias de aprendizaje que deben poner en marcha los profesores y subyace en el fondo una crítica del rol de éstos (enseñanza desenfocada, centrada en los detalles, desmotivadora, pasiva, etc.). Todo ello debería motivar al profesorado a realizar una autocrítica del papel que está desempeñando en la implementación del nuevo currículo. Así, deberían pasar a liderar el proceso, utilizando las estrategias metodológicas más adecuadas para lograr los mejores aprendizajes de nuestros estudiantes. Una manera de proceder podría ser la que se deduce del trabajo de Whittle et al [19], consistente en el análisis de las causas de cada ítem en particular, el planteamiento de posibles soluciones y la aplicación de la considerada más eficaz.

La percepción de los estudiantes en los otros dominios, aunque ha decrecido con respecto a los estudiantes del año 2008, no resulta tan alarmante. La percepción acerca de los profesores (dominio 2) revela un tipo de profesor poco flexible y autoritario que dificulta la relación alumno-profesor, lo cual no es responsabilidad del Plan de Bolonia, y también estos ítems eran del mismo tenor en los estudiantes de primero del 2008. En esta línea se encontraría la alta valoración, similar a la de sus compañeros de currículo tradicional, del ítem 'los docentes ridiculizan a los estudiantes. Por el contrario, en ambas universidades destaca la buena valoración de la preparación del profesorado, acerca del conocimiento de sus materias respectivas y de la claridad de los ejemplos que entregan, aunque la valoración disminuye en la UZar el año 2011 respecto a la del año 2008.

En relación con la autopercepción académica, en la UZar deben destacarse como peor valorados los objetivos que debería cumplir la enseñanza: buena preparación para la profesión, buena base para el trabajo futuro, desarrollo de destrezas para resolver problemas e inteligencia emocional (empatía), reforzando los resultados encontrados en el dominio 1 sobre la percepción de la enseñanza. Los resultados de la UCh en el 2011 se encuentran en línea con los del 2008, con la excepción del ítem 'soy capaz de memorizar todo lo que es necesario'. Es uno de los tres ítems negativos recogidos por Whittle et al [19] en la Universidad de Leeds y por muchos de los trabajos publicados al respecto [20,21,23,31]. Esto apunta a que el volumen de información requiere una reducción en los currículos médicos. Cuestiones como ésta o como la del estrés marcan un claro contraste con las comentadas en el dominio de enseñanza y profesores y no son auténticas críticas, sino que manifiestan la necesidad de los estudiantes de buscar nueva información y respuestas a lo largo de su carrera [19].

Respecto a la percepción del ambiente de aprendizaje, los estudiantes de ambas universidades señalan una baja valoración respecto al ítem 'Los horarios de la Escuela/Facultad están bien programados', obteniéndose la valoración más baja el año 2011 con una media de 1,2 en la UZar y 1,0 en la UCh. Esto 
demuestra que el manejo del tiempo constituye un problema general que se podría asociar al ítem del dominio 1 que señala que 'El tiempo destinado a la enseñanza es bien utilizado' que obtiene valoraciones similares. Otro ítem preocupante en la UZar se refiere a: 'en esta escuela/facultad la copia en las pruebas constituye un problema', ya que constituiría un factor desmotivador, y ante la competitividad existente se deberían abordar dos cuestiones: evaluar si la competitividad es desmesurada y si el control de la limpieza de las pruebas es adecuado.

Respecto a la autopercepción social, los alumnos de ambas universidades obtienen puntuaciones altas, alrededor de 3, en cuatro de los siete ítems de este dominio. Sin embargo, los tres ítems restantes merecen un comentario especial. El ítem 'hay un buen sistema de apoyo para los estudiantes que sufren estrés' obtiene bajas puntuaciones en muchas escuelas/facultades, además de las aquí estudiadas (UZar y UCh) [19]. Es curioso, no obstante, porque en el caso de la UZar y de la UCh existe un gabinete psicológico y un departamento de apoyo a los estudiantes, respectivamente. Resulta decepcionante que habiéndose denunciado [17] no se haya hecho nada al respecto (conocimiento, divulgación, etc.). El ítem 'estoy demasiado cansado para disfrutar los cursos que estoy tomando' aparece con baja puntuación en todos los cursos de la UCh; en cambio, en la UZar, tiene un descenso preocupante en el curso del año 2011. Este resultado sería fuertemente atacado desde el punto de vista empresarial, ya que es piedra angular en el márketing. Sin embargo, la cantidad de estudiantes que desean y solicitan estudiar medicina está por encima de cualquier expectativa y no es necesario atraer a los estudiantes con referencias de este tipo. Por el cansancio invocado y por otras posibles causas, existe un ítem también con pobre puntuación $(1,8)$, 'rara vez me aburro en las clases', que aunque se trata de un ítem que en ninguna exploración ha tenido puntuaciones elevadas, viene a corroborar lo dicho en el anterior. Es muy probable que en la UCh el ambiente social (huelgas, economía, etc.) haya incidido de manera decisiva en algunas percepciones de este dominio.

En conclusión, los resultados negativos encontrados tras la aplicación de la reforma o Plan de Bolonia, especialmente en el dominio de la percepción de la enseñanza, conciernen sólo a la Facultad de Medicina de la UZar. Estos resultados en absoluto ponen en juicio los principios generadores de la reforma del Plan de Bolonia y al replicar este estudio en cualquier otra facultad los resultados podrían ser totalmente distintos y estimuladores. Aparentemente, en la UZar no se estaría aplicando el Plan de Bolonia de la manera más adecuada, de modo que las autoridades y profesores deberían poner atención a las deficiencias observadas y aplicar de forma urgente las medidas que correspondan para corregirlas.

\section{Bibliografía}

1. The Executive Council WFME. International standards in medical education: assessment and accreditation of medical schools' educational programmes. A WFME position paper. Med Educ 1998; 32: 549-58.

2. Genn JM. Curriculum, environment, climate quality and change in medical education -a unifying perspective. Med Teach 2001; 23: 445-54.

3. Roff S, McAleer S. What is educational climate? Med Teach 2001; 23: 333-4.

4. Hammonds SM, O'Rourke M, Kelly M, Bennett D, O'Flynn S. A psychometric appraisal of the DREEM. BMC Med Educ 2012; 12: doi:10.1186/1472-6920-12-2.

5. Genn JM, Harden RM. What is medical education here really like? Suggestions for action research studies of climates of medical education environments. Med Teach 1986; 8: 111-24.

6. Genn JM. Curriculum, environment, climate, quality and change in medical education: a unifying perspective. In Genn $\mathrm{JM}$, ed. Curriculum, environment, climate, quality and change in medical education: a unifying perspective. AMEE Education Guide No. 23. Dundee: Association for Medical Education in Europe; 2001. p. 7-28.

7. Plucker JA. The relationship between school climate conditions and student aspirations. J Educ Res 1998; 91: 240-6.

8. Pimparyon P, Roff S, McAleer S, Poonchai B, Pemba S. Educational environment, student approaches to learning and academic achievement in a Thai nursing school. Med Teach 2000; 22: 359-64.

9. Lizzio A, Wilson K, Simons R. University students' perceptions of the learning environment and academic outcomes: implications for theory and practice. Stud Higher Educ 2002; 27: 27-52.

10. Mayya SS, Roff S. Students' perceptions of the educational environment: a comparison of academic achievers and underachievers at Kasturba Medical College, India. Educ Health 2004; 17: 280-91.

11. Hutchins EB. The 1960 medical school graduate: his perception of his faculty, peers and environment. J Med Educ 1961; 36: 322-9.

12. Pace CR, Stern GG. An approach to the measurement of psychological characteristics of college environments. J Educ Psychol 1958; 49: 269-77.

13. Riquelme A, Fuentes G, Jeria A, Méndez I, Aranís C, Larios G, et al. Ambiente educacional y calidad de la docencia en la escuela de medicina. ARS Med (Santiago) 2007. URL: http:// bases.bireme.br/cgi-bin/wxislind.exe/iah/online/?IsisScript= iah/iah.xis\&src=google\&base $=$ LILACS\&lang=p\&nextAction $=$ lnk\&exprSearch $=511364 \&$ indexSearch=ID.

14. Proceso de Bolonia: definición y características. URL: http:// urbanres. blogspot.com.es/2009/03/proceso-de-boloniadefinicion-y.html.

15. Roff S, McAleer S, Harden RM, Al-Qahtani M, Uddin AA, Deza H, et al. Development and validation of the Dundee Ready Education Environment Measure (DREEM). Med Teach 1997; 19: 295-9.

16. Díaz-Véliz G, Mora S, Bianchi R, Gargiulo PA, Terán C, Gorena D, et al. Percepción de los estudiantes de medicina del ambiente educativo en una facultad con currículo tradicional (UCH-Chile) y otra con currículo basado en problemas (UNC-Argentina). Educ Med 2011; 14: 27-34.

17. Escanero JF, Mora S, Arce J, Bianchi R, Díaz-Véliz G, Gargiulo PA, et al. Estilos de aprendizaje y currículum: propuestas de mejora. Zaragoza: Prensas Universitarias de Zaragoza; 2009 . 
18. Díaz-Veliz G, Escanero JF, Mora S. Estilos, enfoques y contexto de aprendizaje. Escuela de Medicina de la Universidad de Chile. Zaragoza: Prensas Universitarias de Zaragoza; 2011.

19. Whittle S, Whelan B, Murdoch-Eaton DG. DREEM and beyond; studies of the educational environment as a means for its enhancement. Educ Health (Abingdon) 2007; 20: 7.

20. Roff S, McAleer S, Ifere OS, Bhattacharya S. A global diagnostic tool for measuring educational environment: comparing Nigeria and Nepal. Med Teach 2001; 23: 378-82.

21. Bassaw B, Roff S, McAleer S, Roopnarinesingh S, De Lisle J, Teelucksingh S, et al. Students' perspectives on the educationa environment, Faculty of Medical Sciences, Trinidad. Med Teach 2003; 25: 522-6.

22. Al-Hazimi A, Zaini R, Al-Hyiani A, Hassan N, Gunaid A, Ponnamperuma G, et al. Educational environment in traditional and innovative medical schools: a study in four undergraduate medical schools. Educ Health 2004; 17: 192-203.

23. Till H. Identifying the perceived weaknesses of a new curriculum by means of the Dundee Ready Education Environment Measure (DREEM) Inventory. Med Teach 2004; 26: 39-45.

24. Jiffry MTM, McAleer S, Fernando S, Marasinghe RB. Using the DREEM questionnaire to gather baseline information on an evolving medical school in Sri Lanka. Med Teach 2005; 27: 348-52.

25. Brown T, Williams B, Lynch M. The Australian DREEM: evaluating student perceptions of academic learning environments within eight health science courses. Int J Med Educ 2011; 2: 94-101.

26. Makhdoom NM. Assessment of the quality of educational climate during undergraduate clinical teaching years in the College of Medicine, Taibah University. Journal of Taibah University Medical Science 2009; 4: 42-52.

27. Aghamolaei T, Fazel I. Medical students' perceptions of the educational environment at an Iranian Medical Sciences University. BMC Med Educ 2010; 10: doi:10.1186/14726920-10-87.

28. Rotthoff T, Ostapczuk MS, De Brun J, Decking U, Schneider M, Ritz-Timme S. Assessing the learning environment of a faculty: psychometric validation of the German version of the Dundee Ready Education Environment Measure with students and teachers. Med Teach 2011; 33: e624-36.

29. Soltani Arabshahi K, Koohpayehzadeh J, Khamseh ME. Investigation of educational climate in major clinical wards in Iran University of Medical Sciences (IUMS) based on DREEM model. Winter \& Spring 2008; 12: 11-5

30. Herrera C, Pacheco J, Rosso F, Cisterna C, Aichele D, Becker S, et al. Evaluación del ambiente educacional preclínico en seis escuelas de medicina en Chile. Rev Med Chile 2010; 138: 677-84.

31. Al-Hazimi A, Al-Hyiani A, Roff S. Perceptions of the educational environment of the medical school in King Abdul Aziz University, Saudi Arabia. Med Teach 2004; 26: 570-3.

32. De Oliveira Filho GR, Schonhorst L. Problem-based learning implementation in an intensive course of anaesthesiology: a preliminary report on residents' cognitive performance and perceptions of educational environment. Med Teach 2005; 27: 382-4.

33. Zawawi AH, Elzubeir M. Using DREEM to compare graduating students' perceptions of learning environments at medical schools adopting contrasting educational strategies. Med Teach 2012; 34: S25-31.

34. Riquelme A, Oporto M, Oporto J, Méndez JI, Viviani P, Salech F, et al. Measuring students' perceptions of the educational climate of the new curriculum at the Pontificia Universidad Católica de Chile: performance of the Spanish translation of the Dundee Ready Education Environment Measure (DREEM). Educ Health (Abingdon) 2009; 22: 112.

35. McAleer S, Roff S. A practical guide to using the Dundee Ready Education Environment Measure (DREEM). In Genn JM, ed. Curriculum, environment, climate, quality and change in medical education: a unifying perspective. AMEE Education Guide No 23. Dundee: Association for Medical Education in Europe; 2001. p. 29-33.

36. Al-Ayed IH, Sheik SA. Assessment of the educational environment at the College of Medicine of King Saud University, Riyadh. East Mediterr Health J 2008; 14: 953-9.

37. Miles S, Leinster SJ. Medical students' perceptions of their educational environment: expected versus actual perceptions. Med Educ 2007; 41: 265-72.

38. Varma R, Tiyagi E, Gupta JK. Determining the quality of educational climate across multiple undergraduate teaching sites using the DREEM inventory. BMC Med Educ 2005; 5 : doi:10.1186/1472-6920-5-8.

39. Shehnaz SI, Sreedharan J. Students' perceptions of educational environment in a medical school experiencing curricular transition Abraham in Unit Arab Emirates. Med Teach 2011; 33: e37-42.

40. Abraham R, Ramnarayan K, Vindo P, Torke S. Students' perceptions of learning environment in an Indian medical school. BMC Med Educ 2008; 8: doi: 10.1186/1472-6920-8-20.

41. Roff S. The Dundee Ready Educational Environment Measure (DREEM) -a generic instrument for measuring students' perceptions of undergraduate health professions curricula. Med Teach 2005; 27: 322-5. 